\title{
CARACTERIZAÇÃO DE COMPÓSITOS PRODUZIDOS COM POLIETILENO DE ALTA DENSIDADE (HDPE) E SERRAGEM DA INDÚSTRIA MOVELEIRA ${ }^{1}$
}

\author{
Éverton Hillig ${ }^{2}$, Setsuo Iwakiri ${ }^{3}$, Mara Zeni Andrade ${ }^{4}$ e Ademir José Zattera ${ }^{5}$
}

\begin{abstract}
RESUMO - Neste trabalho, realizou-se a caracterização de compósitos confeccionados com polietileno de alta densidade "virgem" (v-HDPE) e diferentes tipos de serragem gerados na indústria moveleira. Os compósitos foram produzidos por extrusão, utilizando-se uma extrusora monorrosca de $35 \mathrm{~mm}$ e temperaturas de 160 ${ }^{\circ} \mathrm{C}, 170{ }^{\circ} \mathrm{C}$ e $180{ }^{\circ} \mathrm{C}$ nas zonas 1,2 e 3 , respectivamente. Foram utilizados resíduos de madeira de pinus (Pinus taeda), de aglomerado, de chapa de fibra de média densidade (MDF) e madeira de eucalipto (Eucalyptus grandis), que foram incorporados ao HDPE “virgem", juntamente com um agente de acoplamento à base de anidrido maléico. Para caracterização física dos compósitos, foram realizadas análises por calorimetria diferencial de varredura (DSC) e por microscopia eletrônica de varredura (SEM). Foram também analisadas as propriedades mecânicas de resistência à tração, flexão estática e resistência ao impacto, conforme as normas ASTM D638, D790 e D256. Verificou-se que todos os tipos de serragem agiram como agentes nucleantes, pois os compósitos apresentaram índices de cristalinidade maiores que o v-HDPE puro, e houve influência do tipo de serragem usada na dispersão das fibras na matriz polimérica. Os ensaios mecânicos indicaram diferenças nas propriedades dos compósitos confeccionados com diferentes tipos de serragem. De maneira geral, a inclusão de serragem de aglomerado e de eucalipto proporcionaram compósitos com maior resistência à flexão e ao impacto que aqueles fabricados com MDF e pinus.
\end{abstract}

Palavras-chave: Compósitos, HDPE e serragem.

\section{CHARACTERIZATION OF COMPOSITES MADE FROM HIGH DENSITY POLYTHYLENE (HDPE) AND FURNITURE INDUSTRY SAWDUST}

\begin{abstract}
This work aimed to carry out a characterization of composites made of high density polyethylene (HDPE) and different types of furniture industry-derived sawdust. The composites were produced by extrusion, using a $35 \mathrm{~mm}$ single-screw extruder, at temperatures of $160^{\circ} \mathrm{C}, 170^{\circ} \mathrm{C}$ and $180^{\circ} \mathrm{C}$ in zones 1,2 and 3 , respectively. Particleboard residues from Pinus taeda sawdust, Medium Density Fiberboard (MDF) and Eucalyptus grandis wood were used and incorporated into 'virgin' HDPE along with a coupling agent of maleic anhydride. Differential Scanning Calorimetry (DSC) and Scanning Electronic Microscopy (SEM) were used to carry out a physical characterization of the composites. Mechanical properties, such as tensile strength, static bending and impact were also analyzed, according to the ASTM. It was verified that all types of sawdust acted as nucleant agents because the composites presented greater crystallization index than the pure v-HDPE, and that the type of sawdust used influenced fiber dispersion in the polymeric matrix. The mechanical tests showed differences in the properties of the composites made from different types of sawdust. Overall, the inclusion of particleboard and Eucalyptus $s p$. wood produced composites with higher resistance to static bending and impact than those made with MDF and Pinus sp.
\end{abstract}

Keyword: composites, HDPE and sawdust.

\footnotetext{
${ }^{1}$ Recebido em 15.01.2007 e aceito para publicação em 20.02.2008.

${ }^{2}$ Departamento de Engenharia Florestal da UNICENTRO, Irati, PR. E-mail: <ehilling@ irati.unicentro.br>.

${ }^{3}$ Departamento de Engenharia e Tecnoligia Florestal da UFPR. E-mail: <setsuo@ufpr.br>.

${ }^{4}$ Departamento de Física e Química da Universidade de Caxias do Sul, RS. E-mail: <mzandrade@ucs.br>.

${ }^{4}$ Departamento de Engenharia Química da Universidade de Caxias do Sul, RS. E-mail: <ajzaterr@ ucs.br>.
} 


\section{INTRODUÇÃO}

A cadeia produtiva madeira e móveis gera significativa quantidade de resíduos que, embora muitas vezes seja aproveitada para alguns fins específicos, pode se constituir em problema de gestão ambiental das empresas. Segundo Hillig et al. (2004), entre os tipos de madeiras usadas na indústria moveleira estão as de pinus e eucalipto e os painéis aglomerados e de chapas de fibras de média densidade (MDF). Em geral, dessa matéria-prima são gerados resíduos que se apresentam, principalmente, na forma de retalhos e serragem e que são utilizados sabendo para geração de energia.

Uma alternativa para aproveitamento dos resíduos de madeira e de seus derivados é a sua utilização em compósito polímero-madeira. Compósitos com polímeros termoplásticos tem tido especial interesse desde a última década, principalmente no Brasil, quando a indústria dos plásticos obteve avanços expressivos em mercado e tecnologia. Wolcott (2003) salientou que esses produtos são competitivos em custos de produção quando comparados com produtos de madeira, desde que se prefira o uso de plásticos reciclados nos compósitos. Embora já sejam produzidos industrialmente em diversas partes do mundo, esse produto ainda carece de confiança do mercado brasileiro, por ser um material relativamente novo e admitir composições diversas.

Compósito é uma mistura física de dois ou mais materiais combinados para formar um novo material útil de engenharia com propriedades diferentes em relação aos componentes puros, podendo ser obtido por combinação de metais, cerâmicas ou polímeros (ASTM, 2001). Tita (1999) fez extensa descrição dos materiais compósitos, observando que são constituídos de um ou mais materiais dispersos numa matriz e chamados de reforço ou carga. Os materiais de reforço podem ser fibras curtas, longas, contínuas, descontínuas e outras, com o objetivo de fornecer resistência. A matriz envolve o reforço, dando forma ao compósito. Abaixo de um comprimento mínimo, as fibras deixam de atuar como reforço para fazer o papel de enchimento ou carga.

O importante num compósito é o efeito sinérgico que se observa no sistema, em que as suas propriedades finais são uma mistura melhorada das propriedades de seus componentes individualmente (CALLISTER JR., 2002). Panshin e Zeeuw (1970) descreveram a madeira como um exemplo natural de compósito, altamente complexo, composto por fibras, principalmente celulose, dispersa numa matriz de lignina e hemicelulose.
Diversos processos de produção podem ser usados para compósitos lignocelulósicos com termoplásticos. Maciel et al. (2004) e Milagres et al. (2006) utilizaram o processo convencional para chapas de madeira aglomerada. Nesses casos, foi formado manualmente um colchão com diferentes misturas de partículas de madeira e termoplásticos que, posteriormente, foi prensado numa prensa de pratos planos. O mais comum, entretanto, é o fato de os processos de mistura e de conformação dos compósitos poliméricos reforçados com fibras celulósicas serem realizados em duas etapas, em que na primeira se faz a incorporação das fibras à matriz e na segunda, um processamento de moldagem do produto final. Diversos tipos de processos podem ser empregados em ambas as fases, mas existe uma tendência, tanto na indústria quanto no desenvolvimento de trabalhos científicos, de se utilizar a extrusão dos materiais em extrusora de dupla-rosca, seguida da injeção da mistura para obtenção do produto final (SAHEB e JOG, 1999).

Raj et al. (1989) salientaram que os polímeros mais utilizados nessas misturas são as poliolefinas polipropileno (PP), polietileno de alta densidade (HDPE) e polietileno de baixa densidade (LDPE), de característica apolar. Como os materiais lignocelulósicos apresentam característica polar, o que diminui sua interação com o polímero, faz-se necessária a presença de um agente de acoplamento, para melhorar a interação entre a matriz polimérica e o agregado.

Youngquist (1995) e Hedenberg e Gatenholm (1995) destacaram a necessidade de se utilizar "fibras" de madeira oriundas de florestas de baixa qualidade e de reciclagem dos resíduos em novos produtos, baseados em matrizes de polímeros termoplásticos. A madeira oferece inúmeras vantagens, como baixo custo e baixa densidade. Embora seu principal problema seja a agregação, alguns estudos apontaram que o emprego desse material na matriz polimérica pode melhorar a "performance" do produto acabado.

Em razão da variedade de espécies de madeira e dos diferentes processos a que são submetidas, as fibras de madeira podem se apresentar com diferentes características físicas e químicas. Tais características podem influenciar as propriedades dos compósitos polímero/madeira produzidos. Stark (1999), num estudo com compósitos de polipropileno e madeira em proporções de $20 \%$ e $40 \%$, respectivamente, verificou que o uso de fibras obtidas de cavacos de madeiras 
de folhosas e de coníferas proporcionou maiores valores médios das propriedades de resistência à tração e de resistência à flexão nos compósitos do que o uso de serragem de madeira, principalmente quando se utilizou maior proporção de madeira. Também observou que o emprego das fibras no lugar da serragem causou menor porcentual de contração do compósito no molde.

Em trabalho semelhante, Stark e Rowlands (2003) estudaram os efeitos de diferentes tamanhos de partículas de serragem de madeira nas propriedades mecânicas de compósitos de polipropileno e madeira e compararam as propriedades desses compósitos com as dos compósitos reforçados com fibras de madeira obtidas por desfibramento termomecânico. Verificaram que a razão de aspecto, e não o tamanho da partícula, teve grande efeito sobre as propriedades de resistência e a dureza dos compósitos.

Maiti e Singh (1986) examinaram diferentes tamanhos de partículas de serragem de madeira e sua influência em compósitos com HDPE, sem o uso de agentes de acoplamento, sendo as amostras extrusadas e submetidas a ensaios de tração. Os resultados indicaram aumento do MOE com o decréscimo do tamanho da partícula de 425 para $180 \mu \mathrm{m}$. Entretanto, todos os compósitos apresentaram menor resistência à tração que o uso do polímero puro. Esses autores consideraram que a diminuição da resistência com o aumento do tamanho da partícula foi devida a uma adesão fraca entre os componentes, desde que nenhum agente de acoplamento tenha sido usado.

Num estudo da influência da espécie de madeira nas propriedades de compósitos madeira-plástico, Wolcott (2003) utilizou serragem de madeira de Pinus sp. e Pseudotsuga menziesii, extrusados com HDPE, aditivos e lubrificantes. Embora a madeira dessas espécies contenha MOE e MOR, à flexão, similares e os compósitos produzidos tenham apresentado a mesma densidade, os compósitos produzidos com Pseudotsuga menziesii exibiram valores de MOE e MOR de $78 \%$ e $76 \%$, respectivamente, dos de MOE e MOE apresentados pelos compósitos com Pinus sp. O referido autor acreditou que esse resultado foi devido à diferença na interação entre madeira e plástico em cada uma das duas espécies, em razão das características da madeira de cada espécie.

Li e Wolcott (2004), estudando as propriedades reológicas de compósitos de HDPE e madeiras de Pinus sp. e Acer sp. e usando um reômetro capilar, concluíram que a viscosidade do material depende do porcentual de madeira e da espécie. O fluxo extensional, no entanto, depende fortemente da porcentagem de madeira e menos da espécie desta.

Dessa forma, este trabalho teve por objetivo caracterizar compósitos confeccionados com HDPE e diferentes tipos de serragem de madeira e derivados usados nas indústrias moveleiras numa extrusora monorosca, bem como, especificamente, verificar a influência do emprego de diferentes tipos de serragem nas propriedades físico-mecânicas dos compósitos.

\section{MATERIAL E MÉTODOS}

\subsection{Material}

Foi utilizado resíduo de madeira de pinus (Pinus taeda), aglomerado, chapa de fibra de média densidade (MDF) e serragem de madeira de eucalipto (Eucalyptus grandis). Os resíduos de pinus e de MDF foram obtidos na forma de serragem, respectivamente das operações de corte com serra-de-fita e com serra circular. Como não foi possível obter serragem pura de eucalipto e de aglomerado, essas serragens foram geradas num moinho de martelos, a partir de cavacos de eucalipto e de retalhos de aglomerados.

A madeira de eucalipto foi obtida numa indústria de celulose e com tamanho variando entre 2 e $4 \mathrm{~cm}$ de comprimento e 1 e $2 \mathrm{~cm}$ de largura. Os retalhos de aglomerados (revestidos) foram coletados numa indústria de móveis da região e seu tamanho variou entre 8 e $12 \mathrm{~cm}$ de comprimento e $2 \mathrm{e} 4 \mathrm{~cm}$ de largura. No Laboratório de Polímeros da Universidade de Caxias do Sul, os retalhos de chapas aglomeradas foram reduzidos a dimensões de aproximadamente $4 \mathrm{~cm}$ de comprimento, numa serra-de-fita, para possibilitar seu processamento no moinho de martelos.

$\mathrm{Na}$ fase matricial dos compósitos foi usado polietileno virgem de alta densidade - v-HDPE (HC 7260 LS), da Ipiranga Petroquímica. As propriedades do v-HDPE são apresentadas no Quadro 1.

Foi também utilizado o agente de acoplamento de nome comercial Polybond 3009 para melhorar a interação entre a fase de fibra e a de matriz. Conforme o fabricante, esse aditivo se trata de um HDPE grafitizado com anidrido maléico, recomendado como agente de acoplamento para vidro, mica, Alumina Trihidratada (ATH), fibras de madeira e outras fibras usadas em

R. Árvore, Viçosa-MG, v.32, n.2, p.299-310, 2008 
compósitos com polietileno. Também é recomendado como compatibilizante para blendas de polietileno com polímeros polares como náilon e EVOH. As propriedades físicas que caracterizam esse produto são apresentadas no Quadro 2.

\subsection{Caracterização dos diferentes tipos de serragem utilizados nos experimentos}

A caracterização dos diferentes tipos de serragem utilizados foi realizada empregando análise granulométrica, análise termogravimétrica (TGA) e termogravimetria derivativa (DTG).

A caracterização granulométrica das amostras de madeira foi realizada utilizando-se peneiras da série Tyler (série fina), com peneiras variando de 8 Mesh Tyler até 400 Mesh Tyler. O ensaio foi executado com o auxílio de um agitador mecânico da Produtest, durante $20 \mathrm{~min}$.

As análises termogravimétricas (TGA e DTG) das amostras de madeira foram realizadas em um equipamento Shimadzu, modelo TGA-50, com taxa de aquecimento de $10{ }^{\circ} \mathrm{C} \cdot \mathrm{min}^{-1}$, sob atmosfera de nitrogênio, com um fluxo de $50 \mathrm{~mL} \cdot \mathrm{min}^{-1}$. As condições de ensaio foram da temperatura ambiente até $900{ }^{\circ} \mathrm{C}$, com as massas das amostras variando entre 10 e $15 \mathrm{mg}$. Foram analisadas três amostras de cada tipo de serragem.

\subsection{Confecção dos compósitos}

A serragem foi incorporada ao HDPE, juntamente com 5\% em peso do agente de acoplamento Polybond 3009, por meio de extrusão com extrusora monorrosca, nas proporções de $20 \%$ e $30 \%$. A mistura de HDPE/ serragem/Polybond e a alimentação da extrusora foram realizadas manualmente. Utilizou-se uma extrusora Seibt, com rosca de $35 \mathrm{~mm}$ e temperaturas de $160{ }^{\circ} \mathrm{C}, 170^{\circ} \mathrm{C}$ e $180^{\circ} \mathrm{C}$, nas zonas 1,2 e 3 , respectivamente, segundo descreveu Grison (2005). A velocidade de rotação foi ajustada em $34 \mathrm{rpm}$, com vazão de aproximadamente $6 \mathrm{~kg} / \mathrm{h}$.
O material extrusado, após o resfriamento em água, foi moído em moinho de facas e acondicionado em sacos plásticos, para o processamento seguinte de injeção dos corpos-de-prova. O material, na forma de grânulos, foi secado em estufa para depois ser levado à injetora.

\subsection{Confecção dos corpos-de-prova}

Os corpos-de-prova foram confeccionados por meio de uma ferramenta de injeção, segundo as normas ASTM D638, D790 e D256 para os ensaios de tração, flexão estática e impacto, respectivamente. Após a confecção, os corpos-de-prova foram condicionados em sala climatizada, na temperatura de $20^{\circ} \mathrm{C}$ e a $50 \%$ de umidade relativa, para realização dos ensaios.

\subsection{Caracterização física dos compósitos}

Esta caracterização foi realizada por meio de calorimetria diferencial de varredura (DSC). As amostras com massas variando entre 9 e $10 \mathrm{mg}$ foram submetidas ao ensaio calorimétrico em um equipamento DSC-50, da Shimadzu, sob atmosfera de nitrogênio, com um fluxo de $50 \mathrm{~mL} \cdot \mathrm{min}^{-1}$. As amostras foram aquecidas da temperatura ambiente até $200^{\circ} \mathrm{C}$, à velocidade de 10 ${ }^{\circ} \mathrm{C} / \mathrm{min}$, e mantidas nessas condições durante aproximadamente $5 \mathrm{~min}$, após o que foram resfriadas a $10^{\circ} \mathrm{C} / \mathrm{min}$ até a temperatura ambiente e mantidas em uma isoterma por $5 \mathrm{~min}$. No segundo ciclo de aquecimento, as amostras foram submetidas às mesmas condições adotadas no primeiro aquecimento. Nessa análise, utilizaram-se índio e zinco metálico, para a calibração da energia e temperatura.

Foram analisados os índices de cristalinidade e a cristalinidade relativa das misturas dos diferentes tipos de serragem, somente na proporção de $30 \%$ de serragem e $70 \%$ de HDPE, com o objetivo de verificar a influência dessa serragem em tal proporção. Foram analisadas três amostras de cada compósitos e do HDPE puro, sendo os índices de cristalinidade submetidos a ANOVAe teste de Tukey, para comparação entre médias.

Quadro 1 - Propriedades do v-HDPE utilizado para confecção dos compósitos Table $1-v-H D P E$ properties used to manufacture composites

\begin{tabular}{|c|c|c|c|}
\hline Propriedade & Norma & Unidade & Valores \\
\hline Densidade & ASTM D $792-98$ & $\mathrm{~g} / \mathrm{cm}^{3}$ & $0,959 \pm 0,003$ \\
\hline MFI $190 / 2,16$ & ASTM D $1238-01$ & $\mathrm{~g} / 10 \mathrm{~min}$ & $8,0 \pm 1,6$ \\
\hline Tensão no escoamento (em placa) & ASTM D $638-02$ & Mpa & 31 \\
\hline Dureza Shore D/15 & ASTM D $2240-03$ & - & 65 \\
\hline Resistência ao impacto IZOD a $23{ }^{\circ} \mathrm{C}$ & ASTM D $256-03$ & $\mathrm{~J} / \mathrm{m}$ & 35 \\
\hline Temperatura de amolecimento Vicat $9,8 \mathrm{~N}$ & ASTM D $1525-04$ & ${ }^{\circ} \mathrm{C}$ & 126 \\
\hline
\end{tabular}

Fonte: Boletim Técnico da IPIRANGA PETROQUÍMICA. 
Quadro 2 - Propriedades físicas do Polybond 3009 Table 2 - Physical properties of Polybond 3009

\begin{tabular}{lcc}
\hline Propriedades & Valores Médios & Método de Determinação \\
\hline Forma física & Pellets & \\
Índice de fluidez $(190 / 2.16)$ & $5 \mathrm{~g} / 10 \mathrm{~min}$ & ASTM D 1238 \\
Densidade $\left(23^{\circ} \mathrm{C}\right)$ & $0,95 \mathrm{~g} / \mathrm{cm}^{3}$ & ASTM D 792 \\
Conteúdo de anidrido maléico & $1 \% \mathrm{em} \mathrm{peso}$ & DSC \\
Temperatura de fusão & $127^{\circ} \mathrm{C}$ & \\
\hline
\end{tabular}

Fonte: Boletim Técnico da CHENTURA CORPORATION.

Para análise da dispersão da serragem na matriz polimérica, realizou-se microscopia eletrônica de varredura (SEM), em microscópio eletrônico marca JEOL, modelo ISM - 5800, do Centro de Microscopia Eletrônica da UFRGS, com ampliações variando entre 200 e 10.000 vezes e $20 \mathrm{KV}$ de energia de feixe incidente. As amostras foram imersas em nitrogênio líquido por $5 \mathrm{~min}$ e fraturadas por impacto. A superfície fraturada foi recoberta por uma camada de ouro e as amostras, coladas em suportes individualizados confeccionados com aço inoxidável, no formato de pequenos cilindros com $1,2 \mathrm{~cm}$ de diâmetro por $1 \mathrm{~cm}$ de altura.

\subsection{Análise dos ensaios mecânicos}

Foi realizado um experimento fatorial, no delineamento inteiramente casualizado, com dois fatores: tipo de serragem e proporção de serragem. Foram usados quatro tipos de serragem (Pinus taeda, Eucalyptus grandis, aglomerado e MDF), em duas proporções (20\% e 30\%), sendo também usado apenas HDPE puro, totalizando nove tratamentos com cinco repetições.

A análise dos resultados das propriedades mecânicas foi realizada por meio de análise de variância (ANOVA). Para comparação entre as médias das propriedades nos diferentes tratamentos, quando estabelecidas as diferenças pela ANOVA, foi realizado o teste de Tukey a $5 \%$ de probabilidade de erro.

\section{RESULTADOS E DISCUSSÃO}

\subsection{Caracterização das serragens utilizadas nos experimentos}

A análise granulométrica dos diferentes tipos de serragens utilizados nos experimentos indica que a distribuição do tamanho das partículas varia de um tipo de serragem para outro. Na Figura 1, apresentase a freqüência média de cada tamanho de partícula para cada tipo de serragem usada.

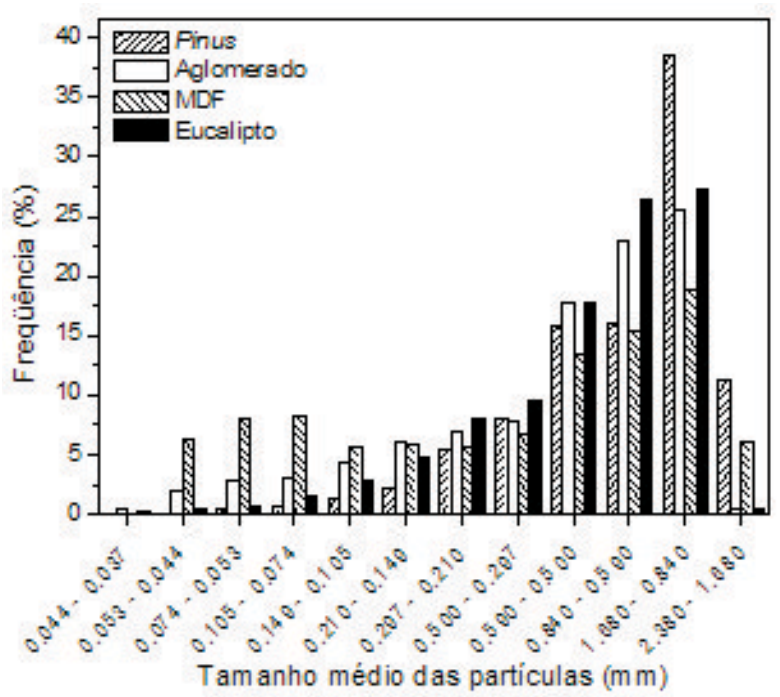

Figura 1 - Distribuição de frequiência do tamanho médio das partículas e dos diferentes tipos de serragens usadas nos experimentos.

Figure 1 - Frequency distribution of the average size of the particles and different types of sawdust used in the experiments.

As serragens de aglomerado e de eucalipto foram geradas no laboratório, a partir de retalhos de chapas aglomeradas e de cavacos de madeira de eucalipto. Esses retalhos e cavacos foram picados em moinho de martelos, produzindo, assim, partículas mais homogêneas em tamanho que se situaram, principalmente, nas classes compreendidas entre $0,210 \mathrm{~mm}$ e $1,680 \mathrm{~mm}$.

As partículas de pinus foram obtidas de uma serraria da região, a partir do desdobro de toras em serra de fita. Por essa razão, a freqüência por tamanho se situa quase toda na faixa entre $0,210 \mathrm{~mm}$ e $2,380 \mathrm{~mm}$, com uma freqüência de cerca de $40 \%$ em apenas uma classe de tamanho $(0,840 \mathrm{~mm}$ a $1,680 \mathrm{~mm})$.

A serragem de MDF é proveniente das indústrias de móveis, a partir das operações de seccionamento,

R. Árvore, Viçosa-MG, v.32, n.2, p.299-310, 2008 
fresamento e lixamento das chapas para obtenção de componentes de móveis. Dessa forma, o uso de diferentes ferramentas acaba por produzir partículas de diferentes tamanhos, o que ocasiona distribuição de freqüência em diferentes classes de tamanho. No entanto, é possível também verificar que a serragem de MDF apresenta maior frequiência nas classes de menor tamanho (abaixo de $0,210 \mathrm{~mm}$ ) e vice-versa, em relação às outras serragens.

Na Figura 2, apresenta-se a perda porcentual de massa das amostras de serragem das madeiras utilizadas em função da temperatura. Nota-se, nessa figura, que as curvas, para cada tipo de serragem, apresentaram-se semelhantes, com faixas de temperatura de perda de massa muito próximas. Entretanto, as perdas de massa de cada tipo de serragem exibiram diferenças, que foram maiores especialmente na fase de estabilização da perda de massa, após a fase de degradação, ou seja, acima de $400{ }^{\circ} \mathrm{C}$.

Visando determinar as temperaturas de início, fim e máximas de decomposição das amostras e permitir caracterizar cada estágio de degradação térmica, foram obtidas as curvas de DTG em função da temperatura, apresentadas na Figura 3. Observa-se, nessa figura, que houve sobreposição de parte dos estágios de degradação, o que foi atribuído ao fato de os diferentes componentes da madeira se degradarem em faixas de temperaturas parcialmente sobrepostas. Além disso, verificou-se que a temperatura inicial dos maiores estágios de perda de massa foi menor nos resíduos de derivados de madeira (MDF e aglomerado), em comparação com os resíduos de madeira, fato que pode estar relacionado aos adesivos que fazem parte desses painéis e a uma possível degradação prévia que sofreu a madeira durante a produção das chapas.

Segundo Gao et al. (2004), a celulose inicia a perda de massa em temperaturas principalmente acima de $300^{\circ} \mathrm{C}$ e mais rapidamente completa seu ciclo de perda até a carbonização, tendo a maior quantidade de voláteis de todos os componentes da madeira. No entanto, a lignina e hemicelulose se decompõem com taxas mais baixas e com maior perda de massa na carbonização. É sabido que, na carbonização da madeira, a hemicelulose é o primeiro composto a se degradar e a lignina, o último.

Bartkowiak e Zakrzewski (2004) comentaram que a lignina é termicamente mais resistente que os carboidratos componentes da madeira. Em temperaturas de até $600^{\circ} \mathrm{C}$, a perda de massa da hemicelulose alcança cerca de $95 \%$; na celulose, esse porcentual fica em torno de $80 \%$, enquanto na lignina não excede $60 \%$.

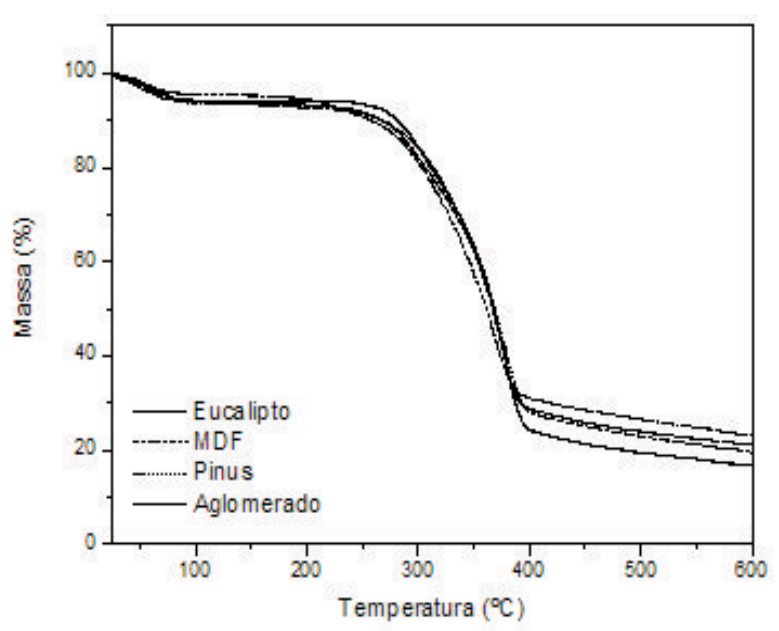

Figura 2 - TGA das amostras dos diferentes tipos de serragem utilizados.

Figure 2-TGA of the samples of the different sawdust types used.

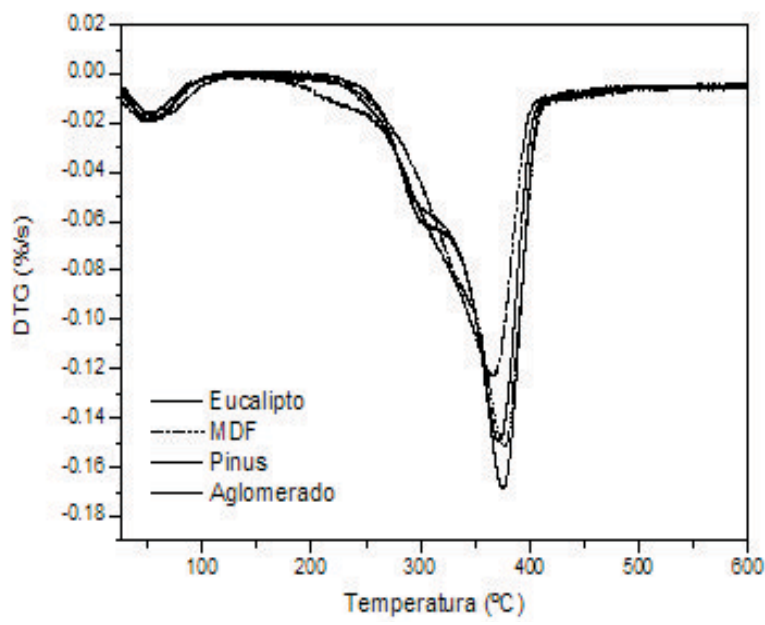

Figura 3 - DTG das amostras dos diferentes tipos de serragem utilizados.

Figure $3-D T G$ of the samples of the different sawdust types used.

Na análise da Figura 3 se observam duas perdas de massa, sendo a primeira em temperaturas pouco acima de $50{ }^{\circ} \mathrm{C}$ referente a água e voláteis da madeira. A segunda perda de massa, com dois picos, um entre $250^{\circ} \mathrm{Ce} 300^{\circ} \mathrm{C}$ e outro entre $350^{\circ} \mathrm{Ce} 450^{\circ} \mathrm{C}$, caracteriza dois estágios de degradação que se referem aos constituintes da madeira, em que o primeiro se refere à degradação da hemicelulose e da celulose e o segundo, à degradação da lignina. 


\subsection{Cristalinidade dos compósitos confeccionados com $30 \%$ de serragem}

No Quadro 3 são apresentadas as entalpias relativas à primeira fusão, segunda fusão e cristalização, juntamente com suas temperaturas. Os valores das entalpias de fusão dos compósitos foram normalizados, em relação à proporção de polímero no compósito, para comparação com HDPE puro.

Segundo Silva et al. (2001), o valor de $\Delta \mathrm{H}^{\circ}$ padrão considerando o polietileno $100 \%$ cristalino fica em torno de $293 \mathrm{~J} / \mathrm{g}$. Assim, esse valor foi usado nos cálculos de cristalinidade relativa.

No polietileno puro foram encontrados um valor da temperatura do pico na cristalização de $114,3^{\circ} \mathrm{C}$ e um $\Delta \mathrm{H}_{\mathrm{c}}$ de $182,6 \mathrm{~J} / \mathrm{g}$. No segundo aquecimento foram observadas uma temperatura de fusão de $132,3{ }^{\circ} \mathrm{C}$ e uma energia $\Delta \mathrm{H}_{c}$ de $180,5 \mathrm{~J} / \mathrm{g}$. O índice de cristalinidade foi calculado em 0,61 .

Nos compósitos, os valores das temperaturas de fusão referentes ao primeiro e segundo ciclos de aquecimento sofreram alterações, em comparação com o HDPE puro. Os valores obtidos no primeiro aquecimento não foram analisados, tendo em vista que sofreram influência da história ocorrida no processamento e que essa influência não foi a mesma todos os compósitos.

As amostras submetidas ao segundo aquecimento reorganizaram suas estruturas e apresentaram variação de entalpia maior. Estatísticamente, os compósitos exibiram índice de cristalinidade maior que o v-HDPE puro, exceto eucalipto. As alterações observadas nas temperaturas de cristalização e segunda fusão podem estar relacionadas com pequenas alterações na estrutura cristalina do v-HDPE. Na Figura 4 está representada a cristalinidade relativa em função do tempo de cristalização. Em geral, as serragens tiveram tendência de agir como carga de reforço e agente nucleante.

\subsection{Microscopia eletrônica de varredura}

Na Figura 5 são apresentadas algumas micrografias da microscopia eletrônica de varredura (SEM) da fratura de amostras dos compósitos confeccionados com os diferentes tipos de serragem. Pode-se verificar, nessa figura, uma influência do tipo de serragem usada na dispersão das fibras na matriz polimérica.

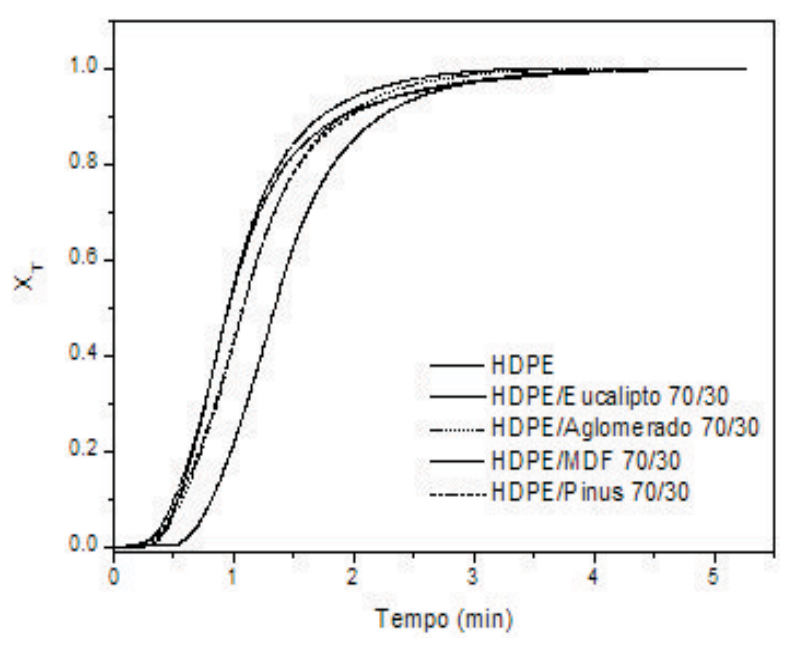

Figura 4 - Índice de cristalinidade relativa dos compósitos confeccionados com $30 \%$ de serragem e com vHDPE puro.

Figure 4-Relative cristallinity index for the composites made with $30 \%$ sawdust and pure $v$-HDEP.

Quadro 3 - Valores de entalpia e de temperatura para a primeira fusão, segunda fusão e cristalização dos compósitos confeccionados com $30 \%$ de serragem

Table 3 - Enthalpy and temperature values for the first fusion, second fusion and crystallization of the composites made with $30 \%$ sawdust

\begin{tabular}{llllll}
\hline Amostras & $\Delta \mathrm{H}_{\mathrm{F} 1}(\mathrm{~J} / \mathrm{g}) \mathrm{T}_{\mathrm{p} 1}\left({ }^{\circ} \mathrm{C}\right)$ & $\mathrm{X}_{\mathrm{CF} 1}$ & $\Delta \mathrm{H}_{\mathrm{C}}(\mathrm{J} / \mathrm{g}) \mathrm{T}_{\mathrm{PC}}\left({ }^{\circ} \mathrm{C}\right)$ & $\Delta \mathrm{H}_{\mathrm{F} 2}(\mathrm{~J} / \mathrm{g}) \mathrm{T}_{\mathrm{p} 2}\left({ }^{\circ} \mathrm{C}\right)$ & $\mathrm{X}_{\mathrm{CF} 2}$ \\
\hline v-HDPE puro & - & - & 182,$6 ; 114,3$ & 180,$5 ; 132,3$ & $0,61 \mathrm{a}$ \\
MDF 30\%/HDPE 70\% & 134,$1 ; 133,3$ & 0,65 & 143,$9 ; 115,6$ & 142,$4 ; 134,4$ & 0,69 \\
Aglomerado.30\%/HDPE 70\% & 123,$2 ; 131,8$ & 0,60 & 152,$6 ; 113,4$ & 150,$5 ; 132,2$ & 0,73 \\
Eucalipto 30\%/HDPE 70\% & 112,$7 ; 133,5$ & 0,54 & 137,$1 ; 115,9$ & 135,$9 ; 135,3$ & $\mathrm{c}$ \\
Pinus 30\%/HDPE 70\% & 116,$9 ; 132,3$ & 0,56 & 144,$1 ; 113,9$ & 142,$4 ; 134,3$ & $0,66 \mathrm{a}, \mathrm{b}$ \\
\hline
\end{tabular}

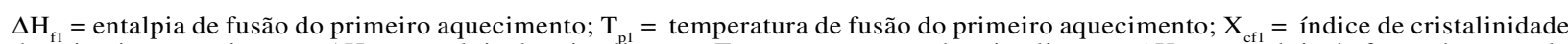
do primeiro aquecimento; $\Delta \mathrm{H}_{\mathrm{c}}=$ entalpia de cristalização; $\mathrm{T}=$ temperatura de cristalização; $\Delta \mathrm{H}_{\mathrm{f}}=$ entalpia de fusão do segundo ciclo de aquecimento; $\mathrm{T}_{\mathrm{p} 2}=$ temperatura de fusão do segundo aquecimento; e $\mathrm{X}_{\mathrm{ct} 2}=$ índice de cristalinidade do segundo aquecimento (médias seguidas por mesmas letras não diferem entre si, pelo teste de Tukey a $5 \%$ de probabilidade de erro). 
HILLIG, E. et al.
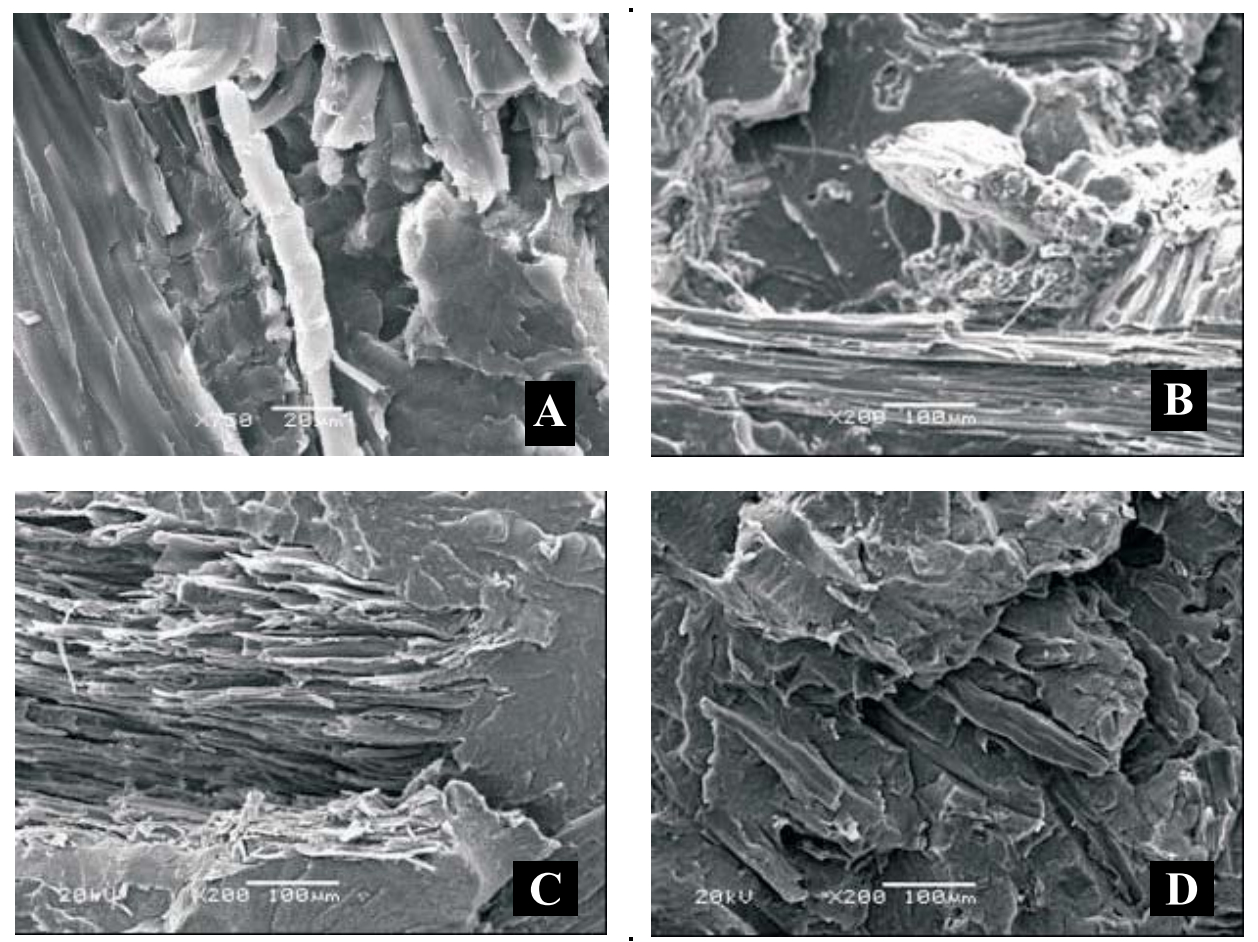

$\mathrm{A}=\mathrm{HDPE} /$ Eucalipto 70/30; $\mathrm{B}=\mathrm{HDPE} /$ Aglomerado 70/30; $\mathrm{C}=$ HDPE/Pinus 70/30; e D = HDPE/MDF 70/30

Figura 5 - Micrografias das superfícies de fratura dos compósitos confeccionados com v-HDPE e diferentes tipos de serragem. Figure 5 -Micrographs of the fracture surfaces of the composites made with different types of sawdust.

Nas serragens de eucalipto (A) e de aglomerado (B) houve a formação de agregados de serragem, que não se dispersaram, sendo esse efeito mais pronunciado na serragem de eucalipto. Nos compósitos confeccionados com pinus (C), verificou-se a existência de alguns agregados, como os que aparecem nessa micrografia, no entanto em menor proporção que nos compósitos com aglomerado e eucalipto. É provável que a ocorrência de agregados esteja relacionada com o tamanho de partícula, visto que nesses três tipos de serragem existe maior freqüência de partículas em classes de tamanho acima de $0,500 \mathrm{~mm}$, consideradas relativamente grandes (ver Figura 1).

Nos compósitos com $30 \%$ de MDF (D) foi possível observar que $\mathrm{o}$ aspecto emaranhado e a existência de agregados de fibras não ocorrem nesse tipo de serragem, que possui maiores freqüências de tamanho de partículas em classes menores (ver item 3.1). Gurram et al. (2002), num trabalho com compósitos confeccionados com termoplásticos (PPe HDPE) e diferentes tipos e tamanhos de partículas fibrosas, verificaram que o aumento do tamanho da partícula de Pinus sp. diminuiu o índice de fluidez do material nos compósitos confeccionados com HDPE. Quanto menor o índice de fluidez, mais difícil a obtenção de uma mistura homogênea, contribuindo para a formação de agregados.

\subsection{Ensaios mecânicos}

No Quadro 4 são apresentados os valores médios das propriedades mecânicas para cada tipo e proporção de serragem usada nos compósitos e também para o v-HDPE puro. Os resultados indicaram que as propriedades mecânicas dos compósitos diferem das do v-HDPE puro e que existem diferenças entre as propriedades dos compósitos confeccionados com diferentes tipos de serragem.

A resistência à tração dos compósitos confeccionados com pinus não diferiu do v-HDPE puro, e os compósitos confeccionados com os outros tipos de serragem diferiram daqueles, mas não entre si, exceto o aglomerado na proporção de $30 \%$. Em relação à proporção de serragem, somente o eucalipto apresentou maior resistência à tração com o aumento da proporção. 
Quadro 4 - Valores médios das propriedades mecânicas dos compósitos confeccionados com diferentes tipos e proporções de serragem e v-HDPE

Table 4-Average values for the mechanical properties of the composites made with different sawdust types and ratios

\begin{tabular}{|c|c|c|c|c|c|c|}
\hline Mistura & Material & $\begin{array}{l}\text { Proporção de } \\
\text { Serragem }(\%)\end{array}$ & $\begin{array}{c}\text { Resistência à } \\
\text { Tração (MPa) }\end{array}$ & $\begin{array}{c}\text { Resistência à } \\
\text { Flexão (MPa) }\end{array}$ & $\operatorname{MOE}(\mathrm{MPa})$ & $\begin{array}{c}\text { Impacto } \\
(\mathrm{J} / \mathrm{m})\end{array}$ \\
\hline 0 & v-HDPE & 0 & $\begin{array}{l}19,36 a \\
(2,18)\end{array}$ & $\begin{array}{l}19,45 a \\
(0,21)\end{array}$ & $\begin{array}{l}568,0 \mathrm{a} \\
(54,3)\end{array}$ & $\begin{array}{l}62,01 \mathrm{~d} \\
(4,88)\end{array}$ \\
\hline 1 & Aglomerado & 20 & $\begin{array}{c}24,37 \mathrm{bc} \\
(0,21)\end{array}$ & $\begin{array}{c}30,63 d \\
(0,37)\end{array}$ & $\begin{array}{c}1095,6 b c \\
(60,1)\end{array}$ & $\begin{array}{c}49,78 b \\
(4,46)\end{array}$ \\
\hline 2 & Aglomerado & 30 & $\begin{array}{c}25,70 \mathrm{c} \\
(0,37)\end{array}$ & $\begin{array}{l}40,15 \mathrm{~g} \\
(0,71)\end{array}$ & $\begin{array}{l}1809,9 d \\
(144,2)\end{array}$ & $\begin{array}{c}53,00 \mathrm{bc} \\
(1,79)\end{array}$ \\
\hline 3 & Eucalipto & 20 & $\begin{array}{c}23,30 \mathrm{~b} \\
(0,56)\end{array}$ & $\begin{array}{c}30,24 d \\
(0,65)\end{array}$ & $\begin{array}{c}1182,0 b c \\
(132,7)\end{array}$ & $\begin{array}{c}68,09 d \\
(7,05)\end{array}$ \\
\hline 4 & Eucalipto & 30 & $\begin{array}{l}25,44 \mathrm{c} \\
(0,65)\end{array}$ & $\begin{array}{l}38,76 f \\
(0,47)\end{array}$ & $\begin{array}{c}1638,3 \mathrm{~cd} \\
(325,9)\end{array}$ & $\begin{array}{c}59,88 \mathrm{~cd} \\
(3,60)\end{array}$ \\
\hline 5 & MDF & 20 & $\begin{array}{c}23,49 b \\
(1,04)\end{array}$ & $\begin{array}{c}28,34 \mathrm{c} \\
(0,82)\end{array}$ & $\begin{array}{l}969,7 b \\
(138,1)\end{array}$ & $\begin{array}{c}37,12 \mathrm{a} \\
(1,90)\end{array}$ \\
\hline 6 & MDF & 30 & $\begin{array}{c}22,76 b \\
(0,57)\end{array}$ & $\begin{array}{l}33,89 \mathrm{e} \\
(0,81)\end{array}$ & $\begin{array}{c}1216,1 b c \\
(93,6)\end{array}$ & $\begin{array}{c}34,77 a \\
(1,62)\end{array}$ \\
\hline 7 & Pinus & 20 & $\begin{array}{l}19,32 \mathrm{a} \\
(0,27)\end{array}$ & $\begin{array}{c}25,58 \mathrm{~b} \\
(0,58)\end{array}$ & $\begin{array}{l}1047,0 \mathrm{~b} \\
(102,5)\end{array}$ & $\begin{array}{l}47,80 \mathrm{~b} \\
(2,99)\end{array}$ \\
\hline 8 & Pinus & 30 & $\begin{array}{c}20,14 a \\
(0,80)\end{array}$ & $\begin{array}{c}28,22 \mathrm{c} \\
(0,39)\end{array}$ & $\begin{array}{c}1382,5 \mathrm{~cd} \\
(51,2)\end{array}$ & $\begin{array}{l}45,91 b \\
(4,54)\end{array}$ \\
\hline
\end{tabular}

NOTAS: Valores médios de cinco corpos-de-prova injetados. Valores entre parênteses referem-se ao desvio-padrão. Médias seguidas de mesmas letras não diferem entre si, pelo teste de Tukey a $5 \%$ de probabilidade de erro.

Os estudos que consideraram os efeitos da proporção de serragem na resistência à tração de compósitos de madeira com termoplásticos apresentam resultados controversos. Robin e Breton (2001) não encontraram relação entre a proporção de serragem de resíduos de spruce e a resistência à tração de compósitos com HDPE, a qual se verificou com valor máximo com $10 \%$ de conteúdo de serragem. Acima desse porcentual, a resistência à tração diminuiu com o aumento na proporção de serragem.

Yamaji (2004) observou que o aumento na proporção de madeira de $10 \%$ para $30 \%$ em compósitos com PP diminuiu a resistência à tração, tanto dos compósitos confeccionados com serragem quanto daqueles feitos com pó-de-lixa. Esse desempenho inferior dos compósitos com maior conteúdo de madeira foi atribuído à maior dificuldade em se obter uma mistura homogênea nas condições experimentais daquele trabalho.

Segundo Correa et al. (2003), embora a fibra de madeira apresente módulo de elasticidade em torno de 20-40 Gpa, as propriedades mecânicas dos compósitos termoplásticos reforçados com "farinha" de madeira tendem a ser inferiores às da madeira in natura. Entretanto, ganhos com a adição de madeira em compósitos, em relação às matrizes termoplásticas puras, são significativos.

Aumento na proporção de serragem elevou a resistência à flexão. Com relação ao tipo de serragem, verificou-se que a de pinus apresentou os valores mais baixos de resistência à flexão, seguida de MDF, eucalipto e aglomerado. Na Figura 6 é apresentado o gráfico dos valores de resistência à flexão dos compósitos em função da proporção de serragem utilizada.

A influência da proporção de madeira na resistência à flexão em compósitos com termoplásticos foi verificada por alguns autores, como Robin e Breton (2001), Hwang et al. (1999) e Fonseca (2005). Nesses trabalhos, os autores constataram que a resistência à flexão cresce com o aumento da proporção de madeira no compósito. Em geral, deve haver uma boa adesão entre as fases de fibra e de matriz para que isso ocorra, mas, mesmo num sistema de baixa adesão, se verifica tal comportamento. Fonseca (2005) considerou, em trabalho, que houve fraca adesão entre as fases de fibra e de matriz. Essa afirmação foi confirmada pelo fato de a resistência à tração dos compósitos diminuir com o aumento da proporção de serragem.

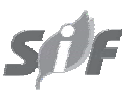

R. Árvore, Viçosa-MG, v.32, n.2, p.299-310, 2008 


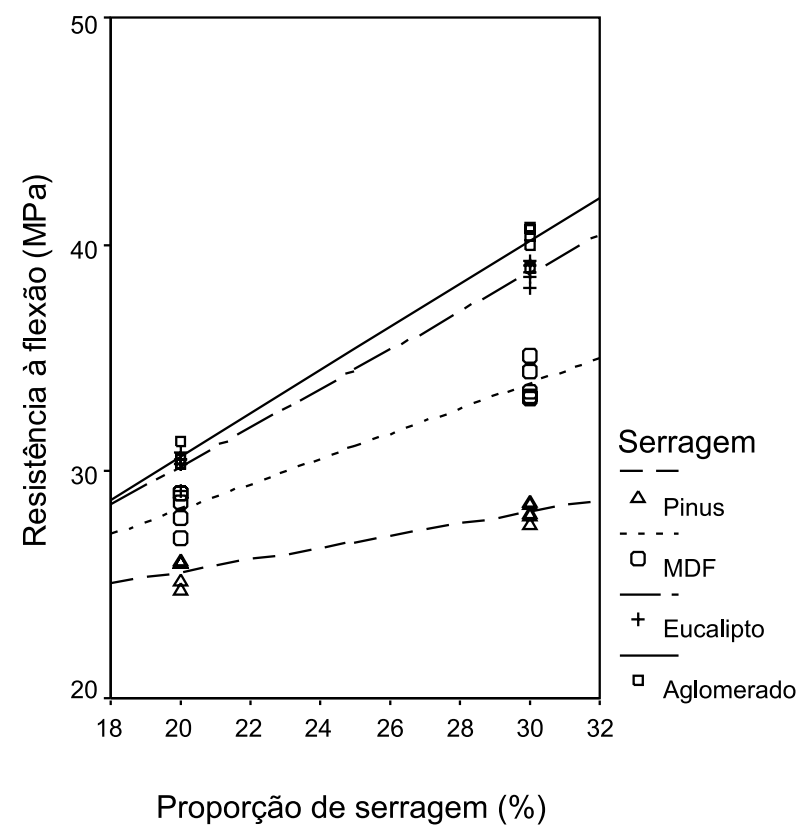

*Linhas de tendência confirmadas pela análise de variância

Figura 6 - Resistência à flexão dos compósitos confeccionados com v-HDPE em função do tipo e proporção de serragem usada.

Figure 6-Resistance to bending of the composites made with $v$-HDPE as a function of the type and ratio of the sawdust used.

No Quadro 4 é possível observar que o MOE da serragem de MDF, na proporção de $30 \%$, foi significativamente inferior ao da serragem de aglomerado. Entretanto, embora não confirmada pela análise estatística, notou-se a tendência de o MOE de MDF e de pinus ser inferior ao do aglomerado e do eucalipto, da mesma forma que a resistência à flexão. Isso indica que o tipo de serragem tem influência significativa na estrutura do material e, em conseqüência, nas suas propriedades, da mesma forma que verificou Wolcott (2003), trabalhando com compósitos plástico de madeira a partir de resíduos de madeira de Pinus sp. e Pseudotsuga menziesii.

$\mathrm{Na}$ análise da resistência ao impacto, com relação ao tipo de serragem, verificou-se que os compósitos apresentaram comportamento diferente. A serragem de MDF teve os menores valores médios, seguida da de pinus e aglomerado, enquanto na serragem do eucalipto os valores médios foram mais altos.

Balasuriya et al. (2001) verificaram diferente resposta na resistência ao impacto de compósitos confeccionados por dois diferentes processos e com HDPE de baixo e médio índices de fluidez. Compósitos extrusados com HDPE de médio índice de fluidez (MMFI) apresentaram maior resistência ao impacto, atribuída a uma melhor distribuição e molhamento das partículas de madeira obtidas pela combinação do processo de extrusão e do índice de fluidez do HDPE. Segundo esses autores, compósitos de MMFI extrusados com até $50 \%$ de proporção de madeira apresentaram faces de fratura típicas no HDPE, em áreas concentradas do polietileno. Tendo em vista que o WPC é um compósito não homogêneo, a fase de matriz nesses compósitos formou uma estrutura em rede contínua que resultou em boa distribuição das partículas e bom molhamento.

Com base nessas afirmações, pode-se inferir que houve melhor distribuição e molhamento das partículas de eucalipto, seguida do aglomerado, pinus e MDF. No entanto, na análise por microscopia de varredura foi observado que os compósitos confeccionados com pinus e MDF tiveram melhor distribuição da serragem de madeira no HDPE, enquanto os compósitos confeccionados com eucalipto e aglomerado apresentaram agregados em determinadas regiões.

Outro fator de influência na resistência ao impacto, embora de menor proporção, é a cristalinidade do compósito. Também nesse caso, verificou-se que não houve comportamento natural do material, pois os compósitos com menor índice de cristalinidade foram os confeccionados com eucalipto, enquanto os de maior índice, os de aglomerado.

Dessa forma, pressupõe-se que a distribuição das partículas deve ter contribuído mais para a resistência ao impacto do que para a adesão na interface e cristalinidade do compósito. De fato, tendo as partículas de aglomerado e de eucalipto granulometria de maior tamanho e estando distribuídas em determinadas regiões do v-HDPE, ou seja, formando agregados, elas proporcionariam maiores espaços entre os agregados de madeira preenchidos com v-HDPE. Isso proporcionaria grande absorção de energia nessas regiões, fazendo que apresentem maior resistência ao impacto.

\section{CONCLUSÕES}

Todos os tipos de serragem agiram como agentes nucleantes, pois os compósitos apresentaram índices de cristalinidade maiores que o v-HDPE puro, exceto o eucalipto. Houve influência do tipo de serragem usada 
na sua dispersão na matriz polimérica, ressaltandose que nos compósitos com eucalipto e aglomerado aparecem agregados que ocorrem em menor proporção nos compósitos com pinus e estão ausentes nos com MDF. Os ensaios mecânicos mostraram diferenças nas propriedades dos compósitos confeccionados com diferentes tipos de serragem que, em parte, foram atribuídas às diversas granulometrias de serragem utilizadas, mas evidenciando influência de outras características inerentes à espécie de madeira. De maneira geral, serragem de aglomerado e eucalipto proporcionou compósitos com maior resistência à flexão e ao impacto que os compósitos fabricados com serragem de MDF e pinus. Com relação à proporção de serragem, verificouse que a resistência à flexão foi maior com o aumento da proporção em todos os compósitos.

\section{AGRADECIMENTOS}

Ao Conselho Nacional de desenvolvimento Científico e Tecnológico-CNPq, à Universidade de Caxias do Sul-UCS e à Universidade Federal do Paraná-UFPR, pelo auxílio financeiro e pelos recursos humanos.

\section{REFERÊNCIAS}

ASTM Standard D3878-01. Standard terminology for composite materials. West Conshohocken: ASTM International, 2001.

ASTM Standard D256-97. Standard test methods for detemining the Izod Pendulum Impact resistance of plastics. Annual book of ASTM standards. v.08.01. West Conshohocken: ASTM International, 2000a.

ASTM Standard D638-99. Standard test method for Tensile properties of plastics. Annual book of ASTM standards. v.08.01. West Conshohocken: ASTM International, 2000b.

ASTM Standard D790-99. Standard test method for Flexural properties of unreinforced and reinforced plastics and electrical insulating materials. Annual book of ASTM standards. v.08.01. West Conshohocken: ASTM International, 2000c.
BALASURIYA, P. W.; MAI, Y. W. Mechanical properties of wood flake-polyethylene composites. Part I: Effects of processing methods and matrix melt flow behaviour. Composites Part A: Applied Science and Manufacturing, v.32, p.619-629, 2001.

BARTKOWIAK, M.; ZAKRZEWSKI, R. Thermal degradation of lignins isolated from wood. Journal of Thermal Analysis and Calorimetry, v.77, p.295-304, 2004.

CALlister Jr., W. D. Ciência e engenharia de materiais: uma introdução. Tradução de Sérgio Murilo Stamile Soares. 5.ed. Rio de Janeiro: LTC - Livros Técnicos e Científicos, 2002.

CORREA, C. A. et al. Compósitos termoplásticos com madeira. Polímeros - Ciência e Tecnologia, v.8, n.3, p.154-165, 2003.

FONSECA, F. M. C. Desenvolvimento e caracterização de compósitos à base de Polietileno de Alta Densidade (PEAD) reciclado e fibras vegetais. 2005. 133f. Dissertação (Mestrado em Engenharia de Materiais) - Universidade Federal de Ouro Preto, Ouro Preto, 2005.

GAO, M.; SUN, C.; ZHU, K. Thermal degradation of wood treated with guanidine compounds in air - Flammability study.

Journal of Thermal Analysis and Calorimetry, v. 75, p. 221-232, 2004.

GRISON, K. Reciclagem de resíduos de medium density fiberboard e poliestireno de alto impacto. 2004. 62f. Trabalho de Conclusão de Curso (Graduação em Tecnologia em Polímeros), Universidade de Caxias do Sul, Caxias do Sul, 2005.

GURRAM, S. et al. Aplication of Biorenewable Fibers in Composites.IONAL CONFERENCE, 2002, Saskatoon. Proceedings... Saskatoon: University of Saskatchewan, 2002.

HENDENBERG, P.; GATENHOLM, P. Conversion of plastic/cellulose waste into composites. I. Model of interphase. Journal of Applied Polymer Science, v.56, p.641-651, 1995.

R. Árvore, Viçosa-MG, v.32, n.2, p.299-310, 2008 
HILLIG, É.; SCHNEIDER, V. E.; PAVONI, E. T. Diagnóstico da geração de resíduos e dos sistemas de gestão ambiental das empresas do pólo moveleiro da serra Gaúcha. In: PÓLO moveleiro da serra Gaúcha: geração de resíduos e perspectivas para sistemas de gerenciamento ambiental. Caxias do Sul: Educs, 2004. 165p.

HWANG CHIN-YIN; HSE, CHUNG-Y.; CHOONG, E. T. Effect of configuration and some processing variables on the properties of wood fiber-polyethylene composites. In: INTERNATIONALCONFERENCEON EFFECTIVEUTILIZATIONOFPLANTATIONTIMBER "TIMBERAND WOODCOMPOSITES FOR THE NEXTCENTURY”, 1999, Chi-Tou, Taiwan.

Proceedings... Chi-Tou, Taiwan: Forest Products Association of R.O.C., 1999. (Bulletin, 16)

LI, T. Q.; WOLCOTT, M. P. Rheology of HDPEwood composites. I. Steady state shear and extensional flow. Composites Part A: Applied Science and Manufacturing, v.35, n.3, p.303-311, 2004.

MACIEL, A. S. et al. Painéis de partículas aglomeradas de madeira de Pinus elliottii Engelm., poliestireno (PS) e polietileno tereftalato (PET). Revista Árvore, v.28, n.2, p.257-266, 2004.

MAITI, S. N.; SINGH, K. Influence of wood flour on the mechanical properties of polyethylene.

Journal of Applied Polymer Science, v.32, p.4285-4289, 1986.

MILAGRES, E. G. et al. Compósitos de partículas de madeira de Eucalyptus grandis, polipropileno e polietileno de alta e baixa densidades. Revista Árvore, v.30, n.3, p.463-470, 2006.

PANSHIN, A. J.; ZEEUW DE, C. Textbook of Wood Technology. New York: McGraw-Hill, 1970. 705p.

RAJ, R.G. et al. Use of wood fibers in thermoplastics: VII. The effect of coupling agents in polyethylene/ wood fiber composites. Journal of Applied Polymer Science, v.37, p.1089-1103, 1989.
ROBIN, J. J.; BRETON, Y. Reinforcement of recycled polyethylene with wood fibers heat treated. Journal of Reinforced Plastics and Composites, v.20, n.14, p.1253-1262, 2001.

SAHEB, D. N.; JOG, J. P. Natural fiber polymer composites: a review. Advances in Polymer Technology, v.18, n.4, p.351-363, 1999.

SILVA, A. L. N. et al. Desenvolvimento de materiais à base de poliolefinas e elastômeros metalocênicos. Polímeros, v.11, n.3, p.135-141, 2001.

STARK, N. M. Wood fiber derived from scrap pallets used in polypropylene composites. Forest Products Journal, v.49, n.6, p.39-46, 1999.

STARK, N. M.; ROWLANDS, R. E. Effects of wood fiber characteristics on mechanical properties of wood/polypropylene composites. Wood Fiber Science, v.35, n.1, p.167-174, 2003.

TITA, V. Análise dinâmica teórica e experimental de vigas fabricadas a partir de materiais compósitos poliméricos reforçados. 1999. $125 \mathrm{f}$. Dissertação (Mestrado emEngenharia Mecânica) - Escola de Engenharia de São Carlos, São Carlos, 1999.

WOLCOTT, M. P. Production methods and platforms for wood plastics. In: NON-WOOD SUBSTITUTES FOR SOLID WOOD PRODUCTS CONFERENCE, 2003, Melbourne.

Proceedings... Melbourne: 2003.

YAMAJI, F. M. Produção de compósito plástico-madeira a partir de resíduos da indústria madeireira. 2004. 182f. Tese (Doutorado em Ciências Florestais)- Universidade Federal do Paraná, Curitiba, 2004.

YOUNGQUIST, J. A. Unlikely partners? The marriage of wood and nonwood materials. Forest Products Journal, v.45, n.10, p.25-30, 1995. 